\title{
Effective thermal conductivity of divided silica xerogel beds
}

\author{
A. Bisson ${ }^{\mathrm{a}, *}$, A. Rigacci ${ }^{\mathrm{a}}$, D. Lecomte ${ }^{\mathrm{b}}$, P. Achard ${ }^{\mathrm{a}}$ \\ ${ }^{a}$ Center for Energy Studies (CENERG), École des Mines de Paris, rue Daunesse, BP 207, Sophia Antipolis 06 904, France \\ ${ }^{\mathrm{b}}$ Powders and Processes Research Center (LGPSD), UMR CNRS 2392, École des Mines d Albi, France
}

\begin{abstract}
Apparent thermal conductivity $(\lambda)$ measurements were made on monolithic silica aerogels and divided low-density xerogels. The objective of the tests on monoliths was to determine the variation of $\lambda$ with the solid content. Additional compressive tests were also made to follow the evolution of bulk modulus with apparent density, in order to further optimize the solid content of xerogel particles. Divided xerogels were characterized under the following conditions: at ambient conditions $(T, P$, and relative humidity of water vapor in air), under partial vacuum (from atmospheric pressure to $4 \times 10^{-7}$ bar) and under different relative humidities. The effect of the divided xerogel granularity on $\lambda$ has been studied by using different size range and distributions. The results of our studies indicate an optimum particle density at $0.15 \mathrm{~g} \mathrm{~cm}^{-3}$, and a decrease in $\lambda$ for samples containing fine particles.
\end{abstract}

\section{Introduction}

The thermal insulating properties of silica aerogels were one of their first attributes to be studied, as their effective thermal conductivity is significantly lower than other standard insulating materials [1]. Their synthesis through an economically feasible process has nevertheless not been achieved, partly because of costs induced by supercritical drying [2]. The possibility to obtain low-density xerogels, by evaporative drying of properly surface-modified gels [3], provides an opportunity to reduce the overall processing cost.

In most applications of silica aerogels as thermal insulants, a divided medium can be used. This form presents some advantages and drawbacks: it is easier to synthesize and to use, especially when spaces to insulate \footnotetext{
535.

* Corresponding author. Tel.: +33 493957 495; fax: +33493957

E-mail address: antoine.bisson@ensmp.fr (A. Bisson).
}

have complex shapes. But if the silica aerogel is used in a too-finely divided state, handling problems arise because of the powdery character. An optimum particle size and distribution must then be determined for these reasons and also to achieve low apparent conductivity $\lambda$.

The objectives of the work presented here are firstly to study the effect of the solid content of the dry gel on $\lambda$ and on mechanical properties. Secondly, we studied at room temperature the respective influence of particle size and distribution, air vacuum, and relative humidity of water vapor in air $(\% \mathrm{RH})$ on the thermal conductivity of divided xerogel beds.

\section{Experimental}

\subsection{Materials}

The silica gels used in this study were made by a twostep acid/base catalysis process, with tetraethoxysilane in isopropanol. Gels that were to be ultimately used in 
the divided state were passed through a $5 \mathrm{~mm}$ screen. An aging step at $60^{\circ} \mathrm{C}$ for $24 \mathrm{~h}$ was then made, followed by surface modification with trimethylchlorosilane at $60^{\circ} \mathrm{C}$ for $24 \mathrm{~h}$.

Divided gels were dried by evaporation of solvent at $80^{\circ} \mathrm{C}$, and monolithic gels were dried in supercritical $\mathrm{CO}_{2}$ following a method previously described [4].

\subsection{Physical measurements}

Two parameters were studied using monolithic samples: apparent thermal conductivity $\lambda$ and bulk modulus $K$. The effective thermal conductivity was measured with two transient methods: hot-wire [5,6] and hot-disk [7]. Generally, transient techniques are only used with opacified silica aerogels [8] while steady-state methods are better fitted for samples that have not been opacified [9]. Previous work on silica aerogels comparable to those reported on in this study showed by comparison between hot-guarded plate and hot-wire measurements that direct radiation within this family of silica aerogel samples is detrimental with regards to the 'low' levels of temperature used [10].

The effective thermal conductivity of monolithic samples was measured on cylindrical plates $(8 \mathrm{~mm}$ thickness and $45 \mathrm{~mm}$ diameter) using the hot-disk method [11]. The probe diameter and heat power were, respectively equal to 9.7 or $14.6 \mathrm{~mm}$ and $0.05-0.1 \mathrm{~W}$, while heating time ranged from 10 to $40 \mathrm{~s}$. The value of $\lambda$ was determined by fitting the thermogram with a unidimensional heat diffusion model [11], Eq. (1). The heat capacity $C_{\mathrm{p}}$ of the samples was calculated from the values of dense silica and air (respectively 0.75 and $1.00 \mathrm{Jg}^{-1} \mathrm{~K}^{-1}$ at $300 \mathrm{~K}$ [12]) by a simple weighting rule [13].

$\Delta T(t, x=0)=\left[F_{0}\left(\frac{1}{\lambda \rho C_{\mathrm{p}}}\right)^{1 / 2}\right] t^{1 / 2}$,

where $\Delta T(t, x=0)$ is the temperature increase at the probe versus time $(\mathrm{K}) ; F_{0}$ is the heat flow density provided by the probe $\left(\mathrm{Wm}^{-2}\right) ; \lambda$ is the sample thermal conductivity $\left(\mathrm{Wm}^{-1} \mathrm{~K}^{-1}\right) ; \rho$ is the apparent density of the sample $\left(\mathrm{g} \mathrm{cm}^{-3}\right) ; C_{\mathrm{p}}$ is the heat capacity $\left(\mathrm{J} \mathrm{m}^{-3} \mathrm{~K}^{-1}\right)$; and $t$ is time (s).

The bulk modulus $K$ was determined by nonintrusive mercury porosimetry, according to the model established by Pirard et al. [14].

The other experiments were made on divided xerogel beds, for which particle size was determined with different screen sizes. For these samples, $\lambda$ was measured using the hot-wire technique [5], with probe length, heat power, and heating time equal, respectively, to $80 \mathrm{~mm}$, $0.05 \mathrm{~W}$, and $180 \mathrm{~s}$. For the measurements, the probe was placed vertically in the center of a $60 \mathrm{~mm}$ diameter and $200 \mathrm{~mm}$ height cylinder, filled by the granular particles, without external load. Unpublished results have shown that hot-disk and hot-wire techniques lead to identical thermal conductivity of monolithic samples.

Evolution of $\lambda$ with interstitial air pressure was performed in a partial vacuum chamber from atmospheric pressure to $4 \times 10^{-7}$ bar. Measurements were made from lower to higher pressures, with a $2 \mathrm{~h}$ stabilization between two successive points. Measurements in humid atmosphere were made in a chamber with a relative humidity imposed by a flow of humid air. To accommodate sorption equilibration times, samples were let at least $72 \mathrm{~h}$ before measurement of the effective thermal conductivity was made, the latter being performed without gas flow.

\section{Results}

\subsection{Apparent thermal conductivity of monolithic aerogels}

To correlate with previously published results [15], the present apparent densities studied ranged from 0.07 to $0.17 \mathrm{~g} \mathrm{~cm}^{-3}$. The values obtained at room temperature and atmospheric pressure with the hot-disk method (Fig. 1) are in good agreement with other work in the literature [16]. A minimum is observed for densities between 0.10 and $0.13 \mathrm{~g} \mathrm{~cm}^{-3}$ and corresponding effective thermal conductivities barely vary around $16 \times 10^{-3} \mathrm{Wm}^{-1} \mathrm{~K}^{-1}$. If one considers the density for which $\lambda$ is $<20 \times 10^{-3} \mathrm{Wm}^{-1} \mathrm{~K}^{-1}$, the density range then extends from 0.09 to $0.16 \mathrm{~g} \mathrm{~cm}^{-3}$.

\subsection{Bulk modulus of monolithic aerogels}

Determination of the bulk modulus $(K)$ was derived from the relation established by Pirard between the sample volume and the mercury pressure applied on it [17]. The associated definition is given in Eq. (2):

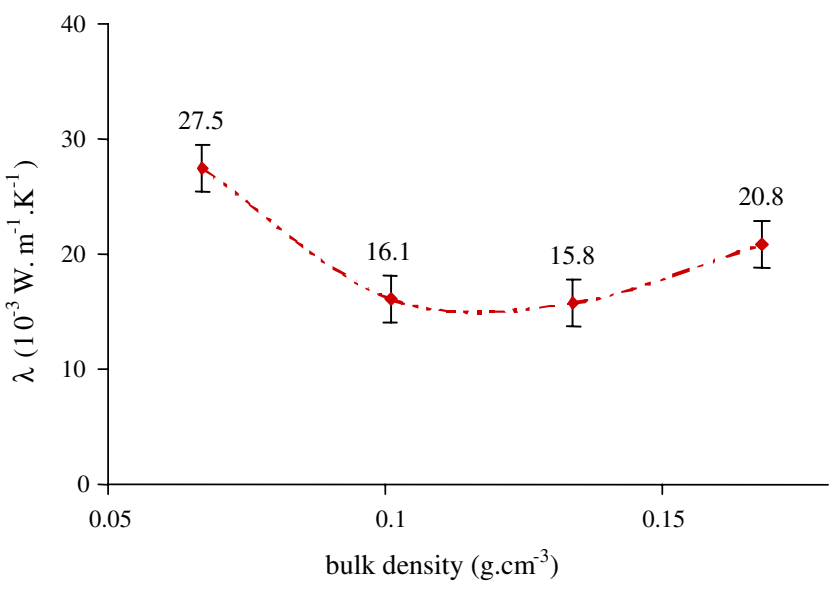

Fig. 1. Apparent thermal conductivity for samples with different apparent densities (dashed line is given as a guide). 


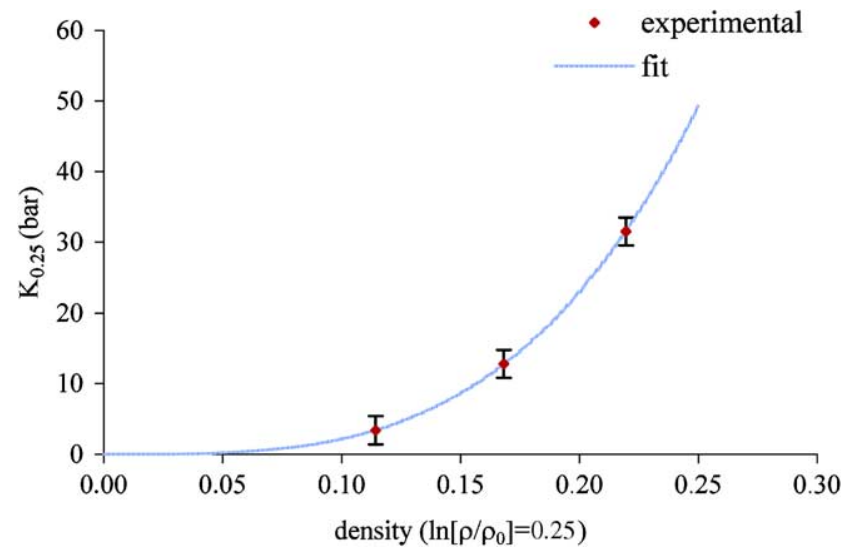

Fig. 2. Bulk modulus (at $\ln \left(\rho / \rho_{0}\right)=0.25$ ) as a function of aerogel apparent density.

$K(V)=V \frac{\mathrm{d} P}{\mathrm{~d} V}$,

where $K$ is the bulk modulus at volume $V$ (bar); $V$ is the sample volume $\left(\mathrm{cm}^{3}\right) ; \mathrm{d} V$ is the variation in volume $\left(\mathrm{cm}^{3}\right)$; and $\mathrm{d} P$ is the variation in the mercury isostatic pressure (bar).

Previous work has shown that for sufficient compression [18], $K$ follows a power law with apparent density $\rho$. The results obtained on samples with different densities enabled determination of the power exponent to be 3.4 (Fig. 2).

\subsection{Particle size distribution and apparent density of granular xerogel beds}

Four different distributions of sample size have been studied (Fig. 3). The apparent density of the xerogel particles is close to $0.15 \pm 0.1 \mathrm{~g} \mathrm{~cm}^{-3}$, and for the particle bed, it decreases to about $0.09 \pm 0.1 \mathrm{~g} \mathrm{~cm}^{-3}$. The interparticle porosity $\varepsilon$ (ratio between void volume and overall volume) is then $\sim 40 \pm 2 \%$, and samples made of finer particles tend to be slightly more compact. The dispersion in size is $1 \mathrm{~mm}$ for three samples, the last one being
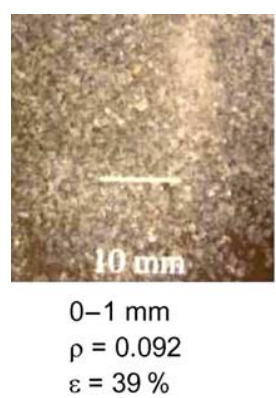

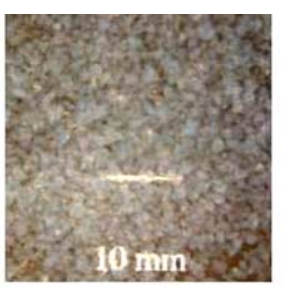

$1-2 \mathrm{~mm}$

$\rho=0.088$

$\varepsilon=41 \%$

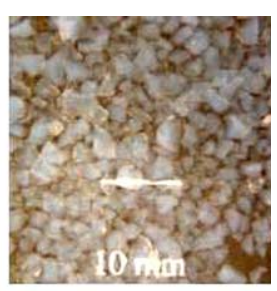

2-3 mm

$\rho=0.087$

$\varepsilon=42 \%$
Fig. 3. Photographs of divided xerogels with their respective apparent density $\rho\left(\mathrm{g} \mathrm{cm}^{-3}\right)$ and interparticle porosity $\varepsilon$.

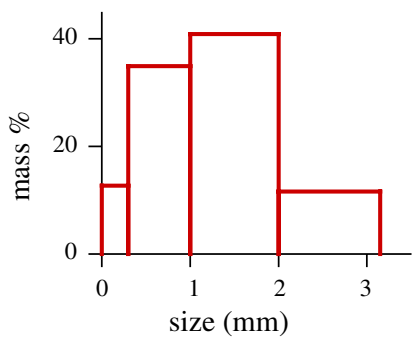

Fig. 4. Particle size distribution of the $0-3 \mathrm{~mm}$ sample.

made of a mix of particle sizes for which the distribution is shown in Fig. 4.

\subsection{Effective thermal conductivity of divided beds at ambient relative humidity and pressure}

The results obtained at atmospheric pressure and ambient relative humidity $(45 \pm 5 \%)$ are given in Fig. 5 . For each particle size and distribution, the sample holder was refilled with xerogel before measuring thermal conductivity. In order to avoid a particulate packing effect on the measurement, values illustrated in Fig. 5 have been obtained by averaging five measurements.

The effect of particle size and distribution on $\lambda$ can clearly be observed from the first three samples as illustrated in Fig. 5. A $20 \%$ drop between $2-3 \mathrm{~mm}$ and $0-1 \mathrm{~mm}$ is observed. This tendency is in good agreement with previous published results [19]. It can also be emphasized that the apparent thermal conductivity obtained for the $0-3 \mathrm{~mm}$ sample appears to be very close to the value obtained for the finest particle size investigated, that is to say the $0-1 \mathrm{~mm}$ sample. The slight decrease observed is not consistent with the evolution of the particle size, and the difference is probably due to experimental error associated with the hot-wire technique.

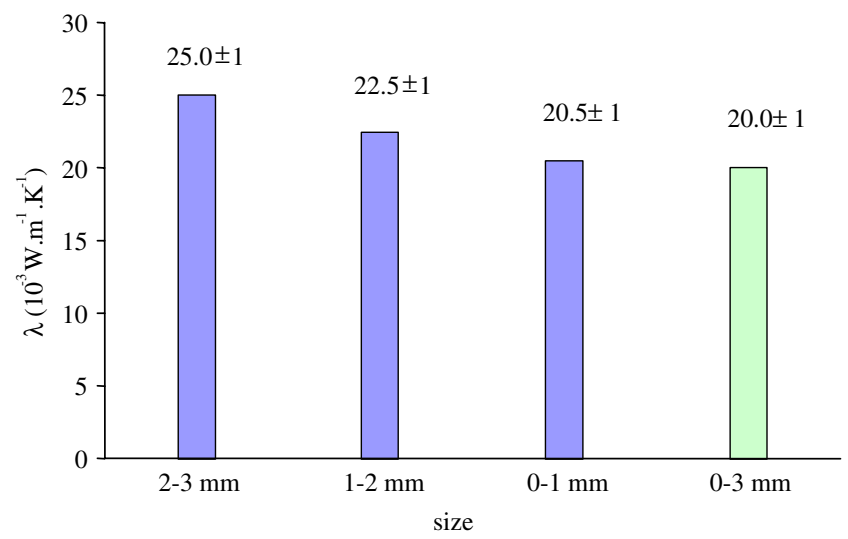

Fig. 5. Apparent thermal conductivity for divided xerogel beds of different particle sizes and distributions $(T=298 \mathrm{~K}$, atmospheric pressure, and $45 \% \mathrm{RH})$. 


\subsection{Effective thermal conductivity of monolith and divided beds at partial vacuum pressure}

Monolithic aerogels and divided xerogel beds have been studied under low partial interstitial air pressure at room temperature. Among the monolithic aerogel families, the density of the investigated sample $\left(0.17 \mathrm{~g} \mathrm{~cm}^{-3}\right)$ was selected to be as close as possible to one of the particulate samples constituting the xerogel beds $\left(0.15 \mathrm{~g} \mathrm{~cm}^{-3}\right)$. The two divided xerogel beds under study were selected to present the lowest investigated apparent thermal conductivity.

If one compares monolithic and divided samples, it can be clearly seen that the thermal conductivity of the monolithic sample is significantly lower than one of the divided beds in the pressure range between atmospheric pressure and $0.2 \mathrm{mbar}$, while the opposite is observed at lower pressures. This very classical result is agrees with previous published results [20].

If one then focuses on the divided samples, it is possible to observe that the two divided media show similar apparent conductivities for $<10$ mbar vacuum whereas the finer sample $(0-1 \mathrm{~mm})$ presents slightly lower apparent thermal conductivities between 10 mbar and atmospheric pressure. This behavior is probably due to the fact that the average size of interparticle voids is a bit smaller because no large particles are present (Fig. 6).

If we now consider that the apparent conductivity of the xerogel bed is a composition of conduction through particles and interparticle voids, then it is possible to use the Knüdsen effect to determine their size. In addition to the experimental curves in Fig. 6, a calculated curve is shown representing the $\lambda$ as function of pressure for air trapped in $0.25 \mathrm{~mm}$ cavities. The values have been calculated using Eq. (3) [21]:

$\lambda_{\mathrm{g}}=\frac{0.026}{1+0.808 \frac{T}{P \delta}}$,

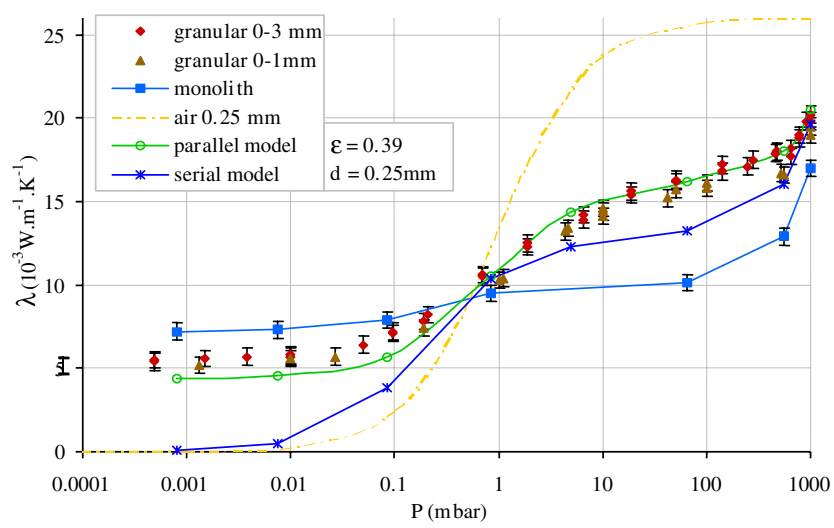

Fig. 6. Apparent thermal conductivity from $4 \times 10^{-4}$ to $1000 \mathrm{mbar}$ for the different samples studied in this work, for air in $0.25 \mathrm{~mm}$ voids (Eq. (3)) and for the calculated values to the serial and parallel models (Eqs. (4) and (5), respectively). where $\lambda_{\mathrm{g}}$ is the apparent thermal conductivity of confined air $\left(\mathrm{Wm}^{-1} \mathrm{~K}^{-1}\right) ; T$ and $P$ are the temperature $(\mathrm{K})$ and pressure (bar), respectively; and $\delta$ is the characteristic size of the cavity $(\mathrm{mm})$.

The inflection point on the curve for the $0.25 \mathrm{~mm}$ cavity is located at the same pressure as on the granular curves. This similarity means that interparticle voids are in this size range. At pressures $<10$ mbar, the diminution in $\lambda$ is due to macropores (see the monolithic sample curve, Fig. 6).

Two classically used Ohm's models [22] have been tested to fit the experimental curves, namely the simple serial and parallel models, Eqs. (4) and (5):

$\lambda_{\text {Serial }}=\frac{\lambda_{\mathrm{p}}}{\varepsilon \frac{\lambda_{\mathrm{p}}}{\lambda_{\mathrm{g}}}+1-\varepsilon}$,
$\lambda_{\text {Parallel }}=\varepsilon \lambda_{\mathrm{g}}+(1-\varepsilon) \lambda_{\mathrm{p}}$,

where $\lambda_{\mathrm{g}}$ is the apparent thermal conductivity of interparticle air $\left(\mathrm{Wm}^{-1} \mathrm{~K}^{-1}\right) ; \lambda_{\mathrm{g}}$ is the apparent thermal conductivity of a xerogel particle $\left(\mathrm{Wm}^{-1} \mathrm{~K}^{-1}\right)$; and $\varepsilon$ is the interparticle porosity.

To determine the values of $\lambda_{\mathrm{g}}$ and $\lambda_{\mathrm{p}}$ used to fit the curves, the following assumptions were made. The interparticle air is supposed to be trapped in $0.25 \mathrm{~mm}$ cavities, so its contribution as a function of pressure is given according to Eq. (3) with $\delta=0.25 \mathrm{~mm}$. The xerogel contribution is taken from the values measured on the monolithic sample assuming that apparent xerogel thermal conductivity is the same in divided and monolithic states. The radiative contribution is contained in the term $\lambda_{\mathrm{p}}$; as determined by Rigacci [23], it should be $\sim 0.002 \mathrm{Wm}^{-1} \mathrm{~K}^{-1}$.

The curves are calculated with experimental interparticle porosity (Fig. 3) and represented in Fig. 6. The serial model does not satisfactorily fit the experimental curves. This lack of fit can be explained by the fact that at low pressures, where gas conductivity is almost zero, effective conductivity does not reduce to the radiative contribution: solid conduction between the particles is present, which is not considered in the serial model. The parallel model better approximates the effective conductivity, because conduction occurs between particles, independently of the fluid phase.

\subsection{Results at different relative humidities}

The dry state of granular gel $(0-1 \mathrm{~mm})$ was obtained after a $24 \mathrm{~h}$ period at $120^{\circ} \mathrm{C}$ to remove adsorbed water. The $\lambda$ was then measured by the hot-wire method to be $20.2 \times 10^{-3} \mathrm{Wm}^{-1} \mathrm{~K}^{-1}$, which is very close to the value in Fig. 5. At $75 \%$ RH (\% relative humidity), $\lambda$ increases to $22.5 \times 10^{-3} \mathrm{Wm}^{-1} \mathrm{~K}^{-1}$, which represents a $10 \%$ rise. But at $100 \% \mathrm{RH}, \lambda$ increases to $33 \times 10^{-3} \mathrm{Wm}^{-1} \mathrm{~K}^{-1}$, a $60 \%$ increase compared to the dry state, whereas the 


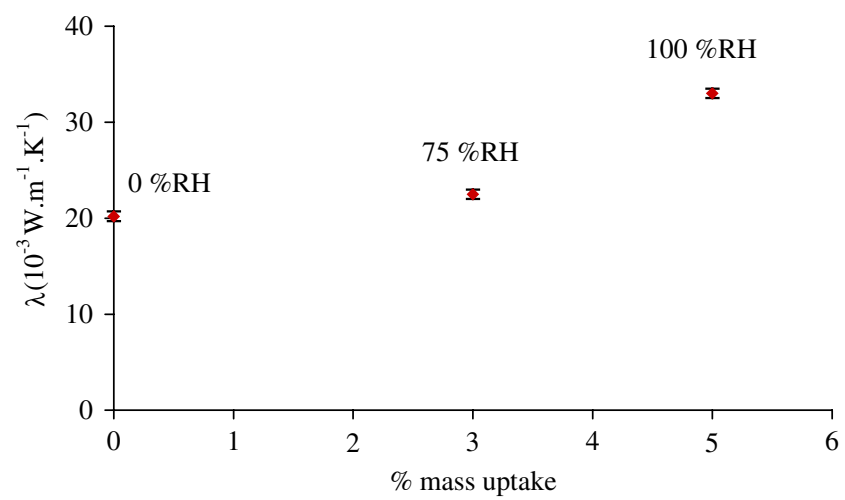

Fig. 7. Apparent thermal conductivity related to the sample mass uptake at different relative humidities.

mass uptake of water in the same time is only $5 \%$ (Fig. 7).

\section{Discussion}

When using the divided xerogel for an application, it is important that the material have mechanical properties such that only minor changes occur in its original characteristics (particle size and distribution, apparent density), especially during handling. That mechanical robustness is why a compromise is necessary between apparent thermal conductivity and mechanical properties. As shown in Fig. 1, an optimum region is observed for low $\lambda$. Concerning the bulk modulus, a monotonic rise is observed as solid content increases, and it can be assumed that it is also the case for the other mechanical properties [24]. The fact that the power-law exponent is high and that the low $\lambda$ region is relatively flat brings us to the following conclusion: when using xerogel particles for thermal insulation, it seems advantageous to choose a silica solid content close to $0.15 \mathrm{~g} \mathrm{~cm}^{-3}$.

The thermal conductivity of a granular medium is influenced by several parameters, such as the volume fraction of each phase and their characteristic dimension [25]. The number and nature of contacts between particles is also influential [20]. In classical granular thermal insulant media, the continuous phase (most generally air) has the lowest conductivity. For low-density xerogels, this situation can be reversed. The objective then is to lower the contribution of air to the total conductivity of the medium, to approach the limiting case of a monolith. From the results presented in Fig. 5, an influence of particle size and distribution is clearly present, but it is difficult to determine which parameter of those described above has the greatest impact. For the $0-1 \mathrm{~mm}$ and $0-3 \mathrm{~mm}$ samples, no discrimination can be made according to the $\lambda$ values, which is not the case for meas-

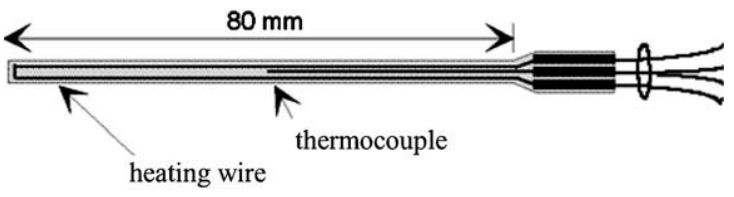

Fig. 8. Schematic of the hot-wire method probe.

urements made under vacuum. The differences between these two particulate size ranges are, however, too small to form definitive conclusions. An interesting observation is that the effective conductivity of the granular xerogel media can be relatively well described by a pure parallel model. If we suppose that this behavior is observed for different sample porosities at ambient pressure, then one can see that the differences observed in Fig. 5 may not be caused by the interparticle porosity, $\varepsilon$, as the $\lambda$ values vary too much to be attributed solely to it.

The characteristic size of interparticle voids that was determined with the partial vacuum measurements cannot be responsible for the differences observed either, as the Knüdsen effect becomes significant at ambient pressure only in voids smaller than $1 \mu \mathrm{m}$ (in this case the apparent air conductivity is $20 \times 10^{-3} \mathrm{Wm}^{-1} \mathrm{~K}^{-1}$ ).

The results of measurements made at three different relative humidities raises the question of metrology, as an artifact could be partially responsible for the large increase observed in saturated atmosphere. The hot-wire method consists of heating a wire and recording the temperature evolution in the proximity of the heated zone (Fig. 8). During heating, endothermic desorption of water occurs on the probe, which acts on the thermocouple as if heat was flowing rapidly. This heat flow results in an overestimated value of $\lambda$, which is more important at elevated relative humidity because for a given heating power, more evaporation can occur. This phenomenon has been described by Langlais [26], and is due to the fact that the measurement is transient and low permeability materials such as aerogels need very long times to reach equilibrium. Steady-state experiments are underway to quantify the evaporation effect.

\section{Conclusion}

The results presented in this work enable us to establish a compromise with respect to the solid content of a xerogel in order to maintain a low thermal conductivity while ensuring sufficient mechanical properties. The influence of the particle size distribution was shown to diminish total conductivity by using fine particles. This effect is not completely explained, but among the controlling parameters, decreased porosity and fewer interparticle contacts are certainly the most important. The results at different relative humidities should be consid- 
ered with caution, as an artifact could be responsible for the large increase in $\lambda$ at saturation.

The presented results in this study can be used to optimize the performances of divided xerogels used as thermal insulating materials in applications such as wall insulation in buildings.

\section{Acknowledgments}

The French Agency for Environment and Energy Management (ADEME) is acknowledged for financial support. The results presented here have been obtained through a collaboration between PCAS and ARMINES/ENSMP/CENERG within contract no. 01.04.062. The authors especially thank their partners in this study: Samira Kherrouf (ADEME), and Pierre Bonnardel and Dominique Valette (PCAS). The authors are also very grateful to Rehé Pirard (University of Liège, Chemical Engineering Laboratory, Belgium) for non-intrusive mercury porosimetry, B. Ladevie and D. Marty (École des Mines d'Albi, UMR CNRS 2392, France) for hot-disk discussions and technical support.

\section{References}

[1] J. Fricke, E. Hümmer, H.J. Morper, P. Scheuerpflug, Rev. Phys. Appl. Colloq. C4 4 (1989) 87.

[2] P.H. Tewari, A.J. Hunt, K.D. Lofftus, Mater. Lett. 3 (1985) 363.

[3] D.M. Smith, R. Deshpande, C.J. Brinker, Mater. Res. Soc. Symp. Proc. 271 (1992) 567.

[4] A. Rigacci, G. Petermann, L. Gullberg, K.I. Jensen, J.M. Schultz, B. Chevalier, P. Nitz, D. Valette, P. Achard, G.M. Pajonk, M.
Durant, M. Ryden, S. Buathier, M.-A. Einarsrud, E. Nilsen, in: Proc. 7th Meeting on Supercritical Fluids, 6-8 December 2000, Antibes, France, 2000, p. 205.

[5] J.J. Healy, J.J. de Groot, J. Kestin, Physica 82C (1976) 392.

[6] H.P. Ebert, V. Bock, O. Nilsson, J. Fricke, High Temp.-High Press 25 (1993) 391.

[7] F.P. Incropera, D.P. DeWitt, Fundamentals of Heat and Mass Transfer, Wiley, 2001.

[8] S.Q. Zeng, A.J. Hunt, W. Cao, R. Greif, J. Heat Transfer 116 (1994) 756.

[9] D. Büttner, R. Caps, J. Fricke, High Temp.-High Press 17 (1985) 375.

[10] A. Rigacci, B. Ladevie, H. Sallee, Br. Chevalier, P. Achard, O. Fufym, High Temp.-High Press. 34 (2002) 549.

[11] S.E. Gustafsson, Rev. Sci. Instrum. 62 (1991) 797.

[12] Handbook of Physics and Chemistry, 83rd ed., CRC, LLC, 2002.

[13] O. Nillson, Å Fransson, O. Sandberg, in: J. Fricke (Ed.), Aerogels, Springer, 1986, pp. 121-126.

[14] R. Pirard, S. Blacher, F. Brouers, J.-P. Pirard, J. Mater. Res. 10 (1995) 2114

[15] L. Hrubesh, R. Pekala, J. Mater. Res. 9 (1994) 731.

[16] E. Hümmer, X. Lu, T. Rettelbach, J. Fricke, J. Non-Cryst. Solids 145 (1992) 211.

[17] R. Pirard, PhD thesis, University of Liége, Belgium, 2000 (in French).

[18] G.W. Scherer, J. Non-Cryst. Solids 186 (1995) 316.

[19] D.M. Smith, A. Maskara, U. Boes, J. Non-Cryst. Solids 225 (1998) 254.

[20] D. Büttner, E. Hümmer, J. Fricke, in: J. Fricke (Ed.), Aerogels, Springer, Berlin, 1986, p. 116.

[21] S.Q. Zeng, A. Hunt, R. Greif, J. Heat Transfer 117 (1995) 758.

[22] R.A. Crane, R.I. Vachon, Int. J. Heat Mass Transfer 20 (1977) 711.

[23] A. Rigacci, PhD thesis, Centre d'Energétique de l'École Nationale Supérieure des Mines de Paris, France, 1998 (in French).

[24] T. Woignier, J. Phalippou, in: Proc. 2nd Int. Symp. on Aerogels, Montpellier, France, 1989, p. 179.

[25] E. Tsotsas, H. Martin, Chem. Eng. Process 22 (1987) 19.

[26] C. Langlais, in: Techniques de l'ingénieur BE 9860, 1995. 